\title{
Transaction
}

\section{Switching Surface Properties of Substrates by Coating with a Cellulose Nanofiber Having a High Adsorbability}

\author{
Ryota Kose, Wakako Kasai, and Tetsuo Kondo
}

\author{
Graduate School of Bioresource and Bioenvironmental Sciences, Kyushu University, \\ 6-10-1 Hakozaki, Higashi-ku, Fukuoka 812-8581, Japan
}

\begin{abstract}
It was reported in our previous paper that the dispersion containing single cellulose nanofibers (= nanocellulose) was prepared from microbial cellulose pellicle using aqueous counter collision (ACC) method [1]. In this study, the nanocellulose has been investigated from the view point of a coating agent. Thus, various substrates such as a filter paper, polyethylene (PE), polyethylene terephthalate (PET), and polypropylene (PP) films or sheets were coated with the nanocellulose, respectively. As a result for a hydrophilic substrate, both water and oil resistances on the surface of the nanofiber-coated filter paper were improved. On the other hand, coating with the same nanocellulose transformed the hydrophobic surface of PE, PET and PP sheets into the hydrophilic. This indicated that the nanocellulose, which was originated from microbial cellulose pellicle, had an effect switching surface properties depending on characteristics of substrates, differing from the wood cellulose nanofibers. Namely, the nanocellulose was preferably coated and adsorbed with the surface of substrates, whereas wood cellulose nanofibers prepared using ACC method and the homogenized pellicle did not accomplish such a behavior. Moreover, the dyeing as well as the improvement in water and oil resistances was performed simultaneously by coating using a mixture of nanocellulose with a dyeing agent.
\end{abstract}

(Received 21 January, 2011 ; Accepted 6 April, 2011)

\section{Introduction}

Gluconacetobacter xylinus (= Acetobacter xylinum) secretes a ribbon-like cellulose nanofiber with ca. $50 \mathrm{~nm}$ in width and $10 \mathrm{~nm}$ in thickness, respectively [2]. The nanofibers are then engaged in a three dimensional network to provide with a gel-like membrane called pellicle, because of random movements accompanied with fiber secretion of the bacteria in a culture medium. To date, the pellicle has been extensively studied to open wider application fields, which are tissue engineering [3,4], electronic device [5], functional foods [6] and medical usage [7]. Most applications above are induced by the unique 3D-network structure constructed with cellulose nanofibers to be employed as a key for the function.

Cellulose is a polysaccharide consisting of $\beta-1,4$ linked glucopyranose skeleton. Both hydrophobic sites due to $\mathrm{C}-\mathrm{H}$ axial bonds and hydrophilic sites due to $\mathrm{C}-\mathrm{OH}$ (hydroxyl) equatorial bonds co-exist in a molecule. Native cellulose fibers assembled with such amphiphilic molecules are supposed to exhibit an amphiphilic property. The hydrophilic site adsorbs water, whereas the hydrophobic site is supposed to capture oils and nonpolar organic solvents such as hexane, toluene and dichloromethane [8].

Recently, the authors proposed Aqueous counter collision (= ACC) $[9,10]$ method to allow bio-based materials to be downsized into nano-objects only using water jets without chemical modification of the molecules. In the previous report [1], ACC treatment could cleave the 3D-network and hierarchical structure of the microbial cellulose pellicle, resulting in aqueous dispersion of fibrillated single nanofibers, which was termed as "nanocellulose". The nanocellulose with an amphiphilic property is considered to be suitable for a coating agent, since the high specific surface areas in the nanofiber were expected to show a high adsorbability to either hydrophilic or hydrophobic materials.

In this study, effects of nanocellulose as a coating agent have been investigated in terms of the following view points : i) it was examined whether oil and water resistances on the surface of substrates may be changed by coating with nanocellulose, and ii) the adsorbability of nanocellulose to either hydrophilic or hydrophobic materials was examined in comparison with wood cellulose nanofibers and the homogenized pellicle. 


\section{Experimental method}

\subsection{Preparation of cellulose nanofibers dispersed in water}

Microbial cellulose pellicle and microcrystalline cellulose powder (Funacel $\mathrm{II}^{\circledR}$ ) were used as starting materials for preparation of cellulose nanofibers.

i) Microbial cellulose nanofibers (= "nanocellulose") [1]

The preparation of the microbial cellulose pellicle and the nanocellulose was followed by the culture and the subsequent $\mathrm{ACC}$ method employed in the previous report [1]. Namely, following inoculated into the SH culture medium [11] in a sterilized plastic container, Gluconacetobacter xylinus $(=$ Acetobacter xylinum: ATCC53582) was cultured statically at $30{ }^{\circ} \mathrm{C}$ to obtain microbial cellulose pellicle. After 2 weeks' incubation, the pellicle with ca. $1 \mathrm{~cm}$ in thickness was established with covering the top of the culture medium. The pellicle was then washed with $0.1 \%$ aqueous $\mathrm{NaOH}$ solution at $80{ }^{\circ} \mathrm{C}$ for 4 hours and successively with water in order to remove protein, bacterial cells and other residues. The purified pellicle was cut into ca. $1 \mathrm{~cm}^{3}$ cubes by a scissors, prior to mixing with deionized water. The mixture containing $0.4 \%(\mathrm{w} / \mathrm{w})$ cellulose fibers was pre-treated using a homogenizer (Physcotron NS-51, Microtec Co., Ltd.) for $5 \mathrm{~min}$ at ca. $20,000 \mathrm{rpm}$. The obtained suspension was provided for ACC treatment using Aqueous counter collision system (Sugino Co.) under 200 MPa of the ejecting pressure with 34 cycle repetition times (Pass) in order to disperse the single cellulose nanofibers with 3-5 $\mu \mathrm{m}$ in length, "nanocellulose", into water [1].

ii) Wood cellulose nanofibers $[9,10]$

The preparation of the single wood cellulose nanofibers was followed by the ACC method employed in the previous report $[9,10]$. Namely, the suspension containing $0.5 \%(\mathrm{w} / \mathrm{w})$ microcrystalline cellulose powder from wood was subjected to ACC treatment under $200 \mathrm{MPa}$ of the ejecting pressure with 30 Pass to let the single nanofibers with 1-2 $\mu \mathrm{m}$ in length disperse into water, as a control.

\subsection{Coating with nanocellulose}

Two kinds of coating methods for a filter paper were performed : i) A filter paper was immersed in an aqueous dispersion containing nanocellulose before dried at $105{ }^{\circ} \mathrm{C}$. ii) The aqueous dispersion was sprayed onto a filter paper before dried. Following repetition of the spraying and drying some times, the paper was finally dried at $40{ }^{\circ} \mathrm{C}$ for 1 hour to be stored in an ambient atmosphere.
In the case of synthetic polymer films, which became more hydrophilic by a corona discharge treatment, such as polyethylene (PE), polyethylene terephthalate (PET), and polypropylene (PP) as a hydrophobic substrate, iii) the aqueous dispersion was sprayed and airdried, and then stored in an ambient atmosphere. iv) After dropped onto the substrate, the dispersion was spread out on the surface using a wire bar. Then, the coated sample was air-dried, and finally dried at $40{ }^{\circ} \mathrm{C}$ for $0.5-1$ hour. The procedure of the dropping and the spreading together with drying were repeated 5 times.

\subsection{Contact angle measurements}

Following a drop of $1 \mu \mathrm{L}$ ultrapure water was put on the surface of the sample to be kept for 1 second, the contact angle was measured using a Drop master DM-300 (Kyowa Interface Science Co., Ltd.) to estimate if the nanocellulose-coated substrate exhibited either hydrophobic or hydrophilic. After all samples were kept at the equilibrium state in an ambient atmosphere with a constant temperature, the contact angles were measured at $25{ }^{\circ} \mathrm{C}$ under less than $40 \%$ of humidity.

\subsection{Scanning electron microscopy (SEM)}

The surface of the filter paper coated with nanocellulose prepared in 2.2 ii) was observed using SEM. Prior to the observation, the sample was mounted on a stab and coated with $\mathrm{Au}$ for SEM observation with JSM5600LV (JEOL Co., Ltd.). The SEM images were acquired as digitalized *.tif files at 8 bit radiometric resolution.

\subsection{Estimation of water and oil resistances for nanocellulose-coated filter papers}

After a drop of mixture of a salad oil (J-OIL MILLS, INC.) with oil red as an indicator (Sudan IV, Tokyo Chemical Industry Co., Ltd.) was put on the coated filter paper prepared in $2.2 \mathrm{i}$ ), both of the front and back sides of the filter paper were apparently observed in order to estimate permeability of the oil into the paper. In the case of water repellency, the blue aqueous indicator (Coomassie Brilliant Blue R-250, Tokyo Chemical Industry Co., Ltd.) was employed. The same procedure as the estimation for the oil resistance was performed.

\section{Results and discussion}

\subsection{Water and oil resistances of filter papers coated with nanocellulose}

Filter papers before and after immersion in an aqueous dispersion containing microbial cellulose nanofibers (= nanocellulose) were compared in terms of the contact angles on the surface. In the case of a non- 
(a)
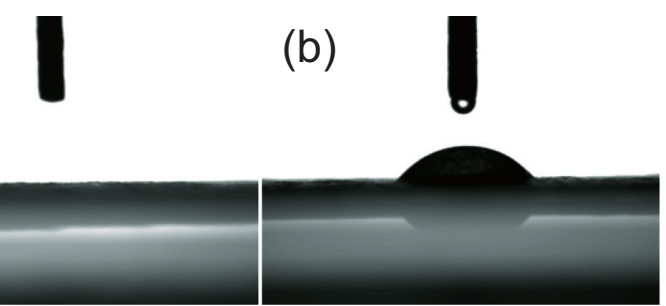

Fig. 1 States of a drop of water on the surface of a filter paper (a) before and (b) after immersed coating with nanocellulose.
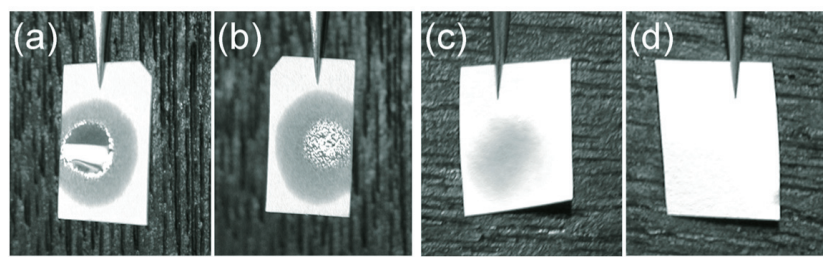

Fig. 2 Front and back sides of filter papers before and after coating with nanocellulose, when a drop of oil containing the indicator was put on the surface. (a) Front and (b) back sides before coating, whereas (c) front and (d) back sides after immersed coating, respectively.

treated filter paper, the contact angle could not be measured as a drop of water soon penetrated into the paper (Fig. 1a). On the other hand, the contact angle of the nanocellulose-coated filter paper exhibited $51^{\circ}$ corresponding to that of a stainless or a silicon-coated glass [12], as the penetration of a drop of water was fairly slow enough to be measured (Fig. 1b). This result indicated that the water resistance was improved by coating with nanocellulose.

In Fig. 2, a drop of oil was put on the surface of filter papers in order to observe penetration of the oil containing a red indicator into the paper. In the case of

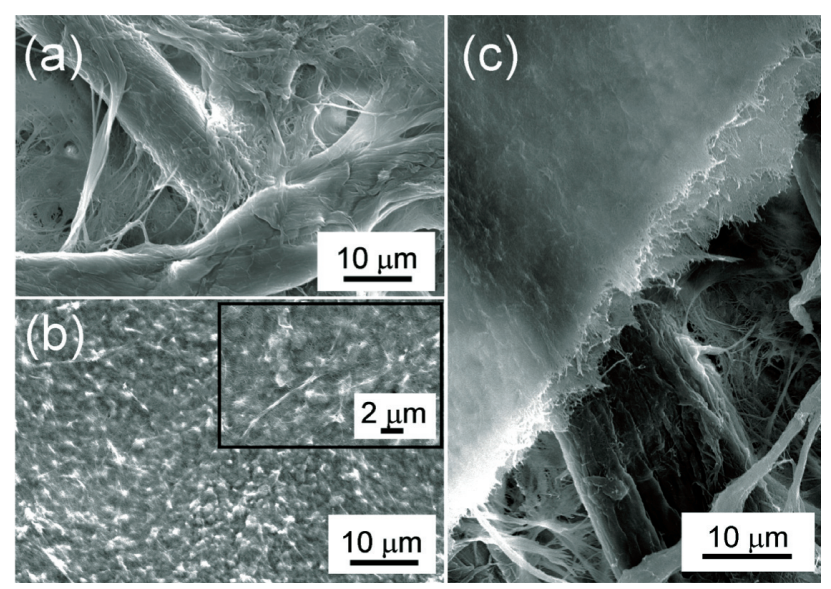

Fig. 5 SEM images of (a) the filter paper before coated and (b) the filter paper spray-coated with nanocellulose. Inset in (b) is the magnified image. Image (c) shows nanocellulose sheets covering over microfibers of a filter paper.

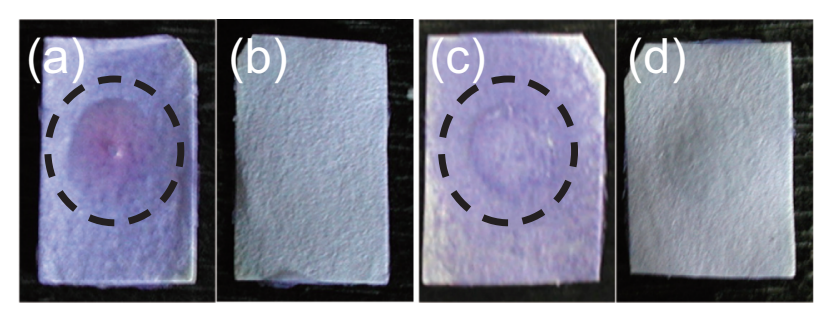

Fig. 4 Front and back sides of the filter papers spraycoated with a mixture of the blue solution and nanocellulose, when a drop of either water or oil was put on the paper. (a) and (b) are front and back sides in oil tests, whereas (c) and (d) are front and back sides in water tests, respectively. The areas surrounded by the dotted lines indicate the spots where (a) oil and (c) water were put, respectively.

non-treated filter paper, the oil was found to penetrate into the sample by observation from the back side of it (Fig. 2b). On the contrary, the surface of the nanocellulose-coated filter paper prevented such penetration, although the oil slightly spread out on the paper (Fig. 2c,d). This indicated that the oil resistance was also improved by coating with the nanocellulose. Fig. 3 shows the surface of the filter paper before (a) and after (b) coated with nanocellulose. Nanocellulose covered roughness due to the deposited microfibers in the filter paper completely by the coating, resulting in the fairly smooth surface at the nano-scale on the filter paper. Indeed, Fig. 3c shows clearly that individual microfibers of the filter paper as the substrate were covered with the smooth nanocellulose sheet on the top.

Taking the above results into consideration, the following reasons are listed for the improvement in both of the resistances. Both sites of hydrophobic due to $\mathrm{C}-\mathrm{H}$ groups and hydrophilic sites due to hydroxyl groups are well known to co-exist in a cellulose molecule, as already described. Native cellulose fibers assembled with such amphiphilic molecules are also assumed to exhibit an amphiphilic property. As the single amphiphilic nanocellulose prepared by ACC has larger specific surface areas due to its fibrillation as well as "nano-size effects", a bundle of the nanofibers could capture a large amount of oil and water. As a result, oil and water are supposed to be kept by the adsorption onto the surface coated with nanocellulose. Further, nanocellulose dispersed in water could coat the surface homogeneously and thereby cover the micropores in the filter papers, resulting in formation of a smooth surface at the nanoscale. Therefore, it is likely to physically prevent the penetration of water as indicated by SEM images of Fig. 3. 
It was noted that the filter papers coated with the mixture of nanocellulose and the blue indicator by the spray method exhibited blue color, and in addition oil and water did not penetrate into it as shown in Fig. 4. Both the dyeing and the improvement of water and oil resistances were simultaneously obtained by coating with the mixture.

\subsection{Adsorbability of nanocellulose}

Nanocellulose, mechanically homogenized microbial cellulose pellicle, and wood cellulose nanofibers were individually sprayed onto the filter paper, before dried. Nanocellulose was preferably adsorbed on the surface of a filter paper, whereas wood cellulose nanofibers formed a film to be peeled from the paper. The homogenized pellicle before ACC treatment, as a control, was not adsorbed strongly enough onto the paper surface when compared with nanocellulose.

The above ability of the three coating agents was examined for the surface of a synthetic polymer film, PET, as a hydrophobic substrate. Fig. 5 shows coated states of nanocellulose and wood cellulose nanofibers on the PET surface after dried. Nanocellulose had the high adsorbability onto the PET surface when compared with wood cellulose nanofibers from microcrystalline cellulose. The high adsorbability of the nanocellulose that was prepared from microbial cellulose pellicle is probably because of i) a favorable entanglement of the longer nanocellulose with a fine structure of the filter paper at the nano-scale $[1,10]$, and ii) the extremely large specific surface areas of the fibrillated nanocellulose provided by ACC treatment.

\subsection{Changing in contact angles by coating of various substrates with nanocellulose}

Fig. 6 shows changing in contact angles on the
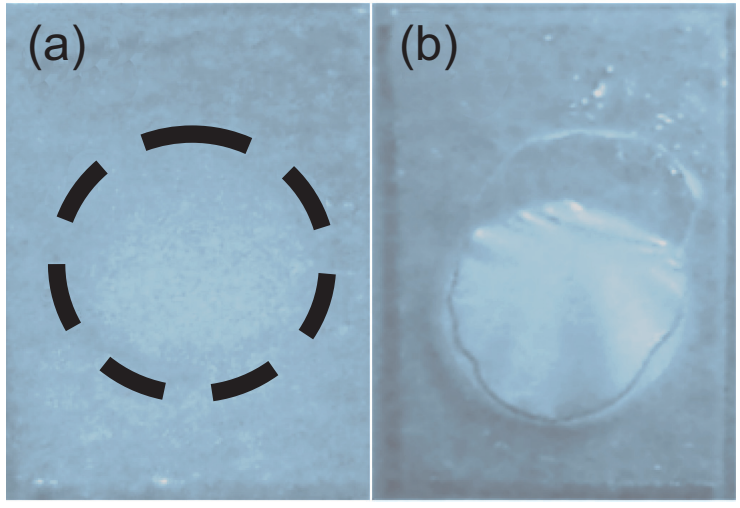

Fig. 5 Coated states on PET surfaces with (a) nanocellulose originated from microbial cellulose and (b) cellulose nanofibers originated from wood, both of which were prepared using ACC treatment. The areas surrounded by the dotted line in (a) is the spot where nanocellulose were adsorbed. surface of PE, PP and PET before and after coated with nanocellulose as described in 2.2 iv). Contact angles on all nanocellulose-coated substrates decreased, indicating that the initial hydrophobic surface became hydrophilic by covering with nanocellulose. All contact angles derived by coating with nanocellulose was smaller than that of a glass [12]. The hydrophilicity did not change for a few hours with a water drop kept on the surface. On the contrary, the contact angles for the hydrophilic surface of a filter paper increased by coating with nanocellulose, as already described in Fig. 1.

Fig. 7 shows the contact angle on PE, PP and PET surfaces depending on repetition times of the spraying with nanocellulose. One cycle of the treatment means the spraying and then air-dried, as described in 2.2 iii). As shown in Fig. 7, the first spraying treatment decreased the contact angle of the surfaces. However, after the second treatment the contact angle exhibited higher than the first one. This behavior in a way confirmed the switching in surface properties of substrates by coating with nanocellulose. In addition, the contact angle after the third spraying tended to be improved more than that for the double coated surface. For some reason, the dense covering repeatedly with the nanocellulose allowed the coated surface to be smoother, which possibly exhibited more hydrophobic. One possible explanation is that the dense covering may cancel the amphiphilicity of the individual nanocellulose more dominantly than the
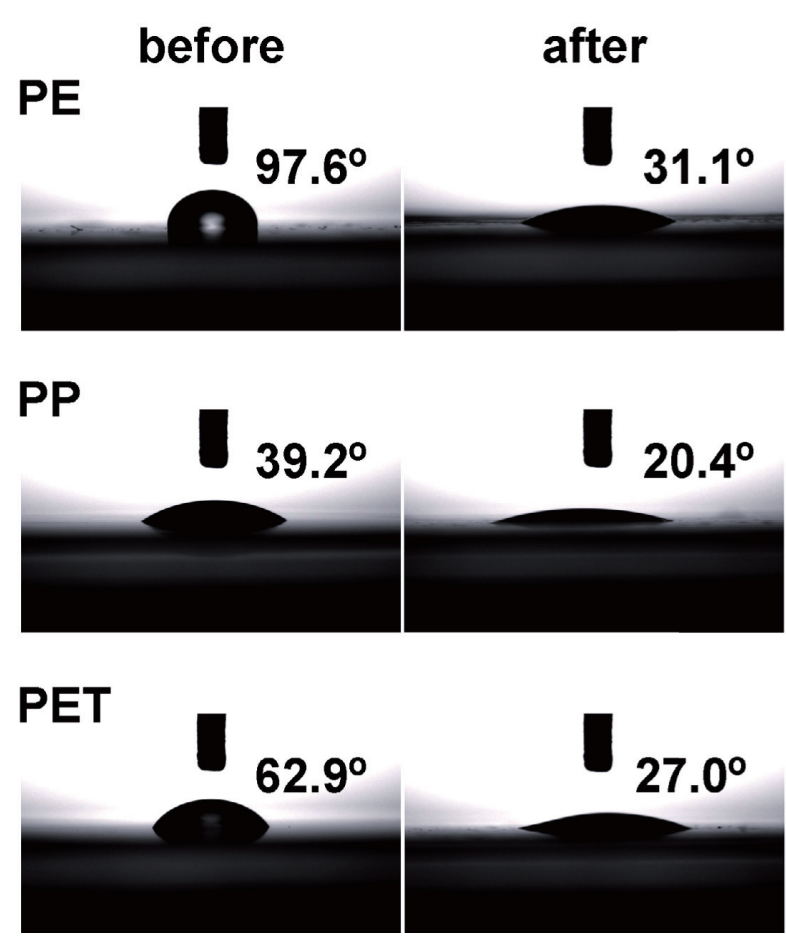

Fig. 6 Contact angles on PE, PP and PET surfaces before and after coated with nanocellulose. 


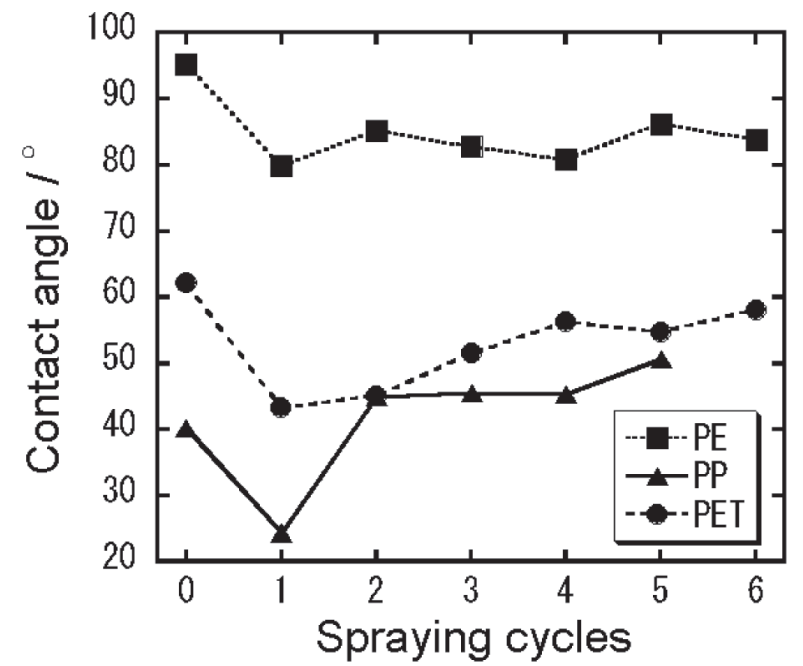

Fig. 7 Contact angles on PE, PP and PET surfaces depending on cycle times of the spraying with nanocellulose.

"switching effect" due to the inherent amphiphilic characteristic of the single nanofiber.

Consideration based on all results above described indicates presumably that, at first, surface properties of the substrates switched from "hydrophobic" to "hydrophilic" or vice versa, by coating with nanocellulose. In the case of hydrophilic substrates, the hydrophilic site of nanocellulose could interact preferably with the surface of substrates to expose the hydrophobic site of the nanofiber into the air side, resulting in switching from the hydrophilic to the hydrophobic on the surface. For the same reason, the opposite switching process due to the nanocellulose could work for the surface of hydrophobic substrates. The once switched surfaces were re-switched by further spraying treatments according to the same mechanism. In the case of PE film, the surface roughness as an RMS value changed at the nanoscale in the range from $100 \mathrm{~nm}$ to $500 \mathrm{~nm}$ depending on the coating times. However, there was not a significant relationship found in between the RMS values and contact angles at each coating time. Tomita et al. [13] reported that the surface roughness could not be necessarily related with the contact angle in pressed and heated microbial cellulose pellicle sheets. In the present study, there was not also found a relationship between the surface roughness and contact angles in the coated surfaces with nanocellulose. However, since it is considered that surface roughness affects the contact angles at a certain range, more detailed research at the nanoscale is required in this aspect.

\section{Conclusions}

Both water and oil resistances of filter papers were improved by coating with nanocellulose that was prepared from the microbial cellulose pellicle. The nanocellulose also exhibited a high adsorbability as a coating agent for the hydrophobic surface of synthetic polymeric materials such as PE, PP, and PET. It was noted that water contact angles on PE, PP and PET films or sheets as a hydrophobic substrate decreased by coating with the nanofibers, whereas those on the nanocellulosecoated filter papers were oppositely improved. The hydrophilic synthetic polymeric surface changed by the first coating was transformed again back to the hydrophobic surface by the further coating. This result indicates that the coating with nanocellulose have provided the switching effect of the surface properties of substrates. It is noted that the adsorbability of nanocellulose prepared using the ACC treatment was found higher than that of wood cellulose nanofibers prepared from the microcrystalline cellulose. Moreover, since the nanocellulose could cover the surface of substrates homogeneously, it is considered suitable as an eco-friendly coating agent. It is also noted that not only improvement in both water and oil resistances of a paper, but dyeing the surface could be performed simultaneously by coating with aqueous dispersions containing ecofriendly nanocellulose mixed with dyes.

\section{References}

1. R. Kose, I. Mitani, W. Kasai, and T. Kondo, Biomacromolecules, 12, 716 (2011).

2. C. Tokoh, K. Takabe, M. Fujita, and H. Saiki, Cellulose, 5, 249 (1998).

3. A. Bodin, S. Concaro, M. Brittberg, and P. Gatenholm, J. Tissue Eng. Regen. Med., 1, 406 (2007).

4. H. Bäckdahl, M. Esguerra, D. Delbro, B. Risberg, and P. Gatenholm, J. Tissue Eng. Regen. Med., 2, 320 (2008).

5. J. Shah and R. M. Brown, Jr, Appl. Microbiol. Biotechnol., 66, 352 (2005).

6. K. -W. Lin and H. -Y. Lin, J. Food Sci., 69, SNQ 107 (2004).

7. D. Klemm, D. Schumann, F. Kramer, N. Heßler, M. Hornung, H. -P. Schmauder, and S. Marsch, Adv. Polym. Sci., 205, 49 (2006).

8. C. Yamane, T. Aoyagi, M. Ago, K. Sato, K. Okajima, and T. Takahashi, Polym. J., 38, 819 
(2006).

9. T. Kondo, M. Morita, K. Hayakawa, and Y. Onda, United States Patent 7, 357, 339 (2005).

10. T. Kondo, R. Kose, H. Naito, A. Takada, and W. Kasai, Colloid Polym. Sci., submitted.
11. S. Hestrin and M. Schramm, Biochem. J., 58, 345 (1954).

12. F. W. Hyde, M. Alberg, and K. Smith, J. Ind. Microbiol. \& Biotech, 19, 142 (1997).

13. Y. Tomita, T. Tsuji, T. Kondo, Sen 'I-Gakkaishi, 65, 73 (2009). 